\title{
Bioimpedancia vectorial y espectroscópica: valoración del estado de hidratación con ambos métodos en hemodiálisis
}

\author{
Ruth Ros Nogales, Mª Teresa Manso Gil, Gema Palacios García, Jacoba Rodríguez Peña
}

Enfermeras Servicio de Nefrología (Unidad de Hemodiálisis). Hospital Universitario Ramón y Cajal. Madrid

\section{Resumen}

EI análisis de bioimpedancia es una herramienta útil para determinar el estado nutricional y de hidratación del enfermo en hemodiálisis. Los dos sistemas más utilizados son la bioimpedancia de monofrecuencia vectorial y la bioimpedancia multifrecuencia espectroscópica que utilizan diferentes criterios para clasificar el estado de hidratación del enfermo dializado. El sistema de bioimpedancia de monofrecuencia vectorial utiliza una escala ordinal de 7 puntos que va desde el valor 3 (mayor hidratación) hasta el valor -3 (mayor deshidratación) definiendo la hiperhidratación cuando el vector está en el eje de hidratación por debajo de la elipse de tolerancia del $75 \%$ (valores 3 y 2 de la escala ordinal). El sistema de bioimpedancia multifrecuencia espectroscópica emplea dos criterios para definir el estado de hiperhidratación prediálisis: $\mathrm{OH}$ superior a 2,5 litros o mayor del $15 \%$ del volumen de agua extracelular.

El objetivo del presente trabajo fue analizar el grado de concordancia entre ambos sistemas de bioimpedancia para definir el estado de hiperhidratación en enfermos tratados con hemodiálisis.

Se llevó a cabo un estudio transversal en 54 enfermos tratados con hemodiálisis a los que se les realizó un análisis de bioimpedancia con monitores de monofrecuencia vectorial y multifrecuencia es-

Correspondencia:
Jacoba Rodriguez Peña
Servicio de Nefrología
Hospital U. Ramón y Cajal
Carretera de Colmenar Viejo Km 9,100, 28033, Madrid
E-mail: Jacoba.rodriguez@salud.madrid.org

pectroscópica inmediatamente antes de una sesión de hemodiálisis.

El grado de equivalencia en la definición de hiperhidratación entre ambos monitores fue mejor cuando se utilizó, en el monitor de multifrecuencia, el criterio de $\mathrm{OH}>15 \%$ del agua extracelular (índice kappa 0.81 , concordancia excelente) que cuando se utilizó el criterio de $\mathrm{OH}>2,5$ litros (índice kappa 0.71 , concordancia aceptable).

Conclusiones: Hay una buena relación entre la escala vectorial de hidratación y el valor $\mathrm{OH}$ (exceso de hidratación). Los criterios utilizados por los monitores de monofrecuencia vectorial y multifrecuencia espectroscópica para definir los estados de hiperhidratación prediálisis tienen una equivalencia aceptable y sus resultados pueden ser intercambiables.

\section{PALABRAS CLAVE:}

- BIOIMPEDANCIA

- VECTORIAL

- ESPECTROSCÓPICA

- HIPERHIDRATACIÓN

Bioimpedance vector and spectroscopic analysis: assessing hydration status using both methods in haemodialysis

\section{Abstract}

Bioimpedance analysis is a useful tool for determining the nutritional and hydration status of haemodialysis patients. The two most commonly used systems are bioimpedance vector single frequency analysis and bioimpedance spectroscopic multiple frequency 
analysis, which use different criteria to classify the hydration status of the dialysed patient. The single frequency bioimpedance vector system uses a scale of 7 points ranging from a value of 3 (most hydration) to a value of -3 (most dehydration), defining hyperhydration when the vector is on the hydration axis below the tolerance ellipse of $75 \%$ (values 3 and 2 on the scale). Multi-frequency spectroscopy uses two criteria to define the pre-dialysis state of hyperhydration: $\mathrm{OH}$ more than 2.5 litres or more than $15 \%$ of the extracellular water volume.

The aim of this study was to analyse the degree of concordance between the two systems of bioimpedance in defining the state of hyperhydration of haemodialysis patients.

A transversal study was carried out on 54 patients undergoing haemodialysis, performing a bioimpedance analysis with single frequency vector and multi-frequency spectroscopic monitors immediately before a haemodialysis session.

The degree of equivalence in the definition of hyperhydration between the two monitors was greater when the criterion of $\mathrm{OH}>15 \%$ of extracellular water was used in the multi-frequency monitor (kappa index 0.81, excellent concordance) than when the criterion of $\mathrm{OH}>2.5$ litres was used (kappa index 0.71 , acceptable concordance).

Conclusions: There is a good relationship between the hydration vector scale and $\mathrm{OH}$ value (excess hydration). The criteria used by the single-frequency vector and multi-frequency spectroscopic monitors show acceptable equivalence and their results can be interchangeable.

\section{KEY WORDS:}

- BIOIMPEDANCE

- VECTOR

- SPECTROSCOPIC

- HYPERHYDRATION

\section{Introducción}

El análisis de la bioimpedancia nos permite cuantificar los diversos compartimentos del cuerpo humano, mediante una técnica no invasiva y de fácil manejo.
Proporciona información útil para valorar la nutrición y la hidratación del paciente sometido a hemodiálisis, aportando a la enfermería una herramienta muy útil para optimizar los cuidados del mismo y mejorar así la educación para la salud. La aparición de monitores sencillos y de precio asequible ha generalizado su uso en los Servicios de Nefrología. Una muestra de ello es el número relevante de comunicaciones relacionadas con la bioimpedancia que se han presentado en los tres últimos Congresos Nacionales de la especialidad.

El monitor de bioimpedancia obtiene los parámetros eléctricos del cuerpo humano (resistencia, reactancia y ángulo de fase) y calcula volúmenes y masas corporales con ecuaciones de predicción que incluyen los datos eléctricos y otras variables como peso, talla, edad y sexo. Estas ecuaciones son distintas para cada monitor, la mayoría solo incluye al componente de resistencia y en muchas ocasiones son difíciles de conocer $(1,2,3,4,5,6)$.

La bioimpedancia multifrecuencia espectroscópica (BIS) y la bioimpedancia monofrecuencia vectorial (BIVA) son los dos sistemas de bioimpedancia más utilizados en España ${ }^{(7,8)}$. En estudios comparativos se ha constatado que los valores de los diversos compartimentos corporales proporcionados con los dos sistemas son diferentes, con una variabilidad intermétodo que impide

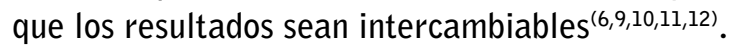

Un objetivo del presente trabajo es analizar si la variabilidad intermétodo es debida a la diferente lectura de las variables bioeléctricas realizadas por los monitores 0 a las ecuaciones utilizadas por cada uno de ellos para el cálculo de los volúmenes y masas corporales. Otro objetivo es comprobar si a pesar de la variabilidad intermétodo, la clasificación de los estados de hidratación definidos por ambos monitores es concordante. El estudio fue realizado en pacientes con enfermedad renal crónica en estadio 5 tratados con hemodiálisis.

\section{Material y métodos}

Estudio transversal realizado en 54 enfermos tratados con hemodiálisis periódica a los que se les hizo un análisis de bioimpedancia con los sistemas BIS y BIVA. Son 36 varones y 18 mujeres, con edad de $69 \pm 14$ años (rango 34-92). Todos ellos eran enfermos estables desde el punto de vista clínico, sin síntomas o signos de insuficiencia cardiaca. El índice de masa corporal era de 26,5 $\pm 3,9$ (rango: 18,3 - 38,3; intervalo de confianza: $25,5-27,6)$. El análisis de bioimpedancia se hizo antes 
de la sesión de hemodiálisis, con el enfermo en decúbito supino, colocando los dos pares de electrodos en muñeca y tobillo del hemicuerpo libre de acceso vascular siguiendo el procedimiento habitual. Primero se realizó la medición en sistema BIVA que utiliza una frecuencia de $50 \mathrm{kHz}$ (monitor EFG ElectroFluidGraph analyzer, Akern SRL, Florence, Italy) y a continuación con el sistema BIS (monitor BCM Fresenius Medical Care, Bad Homburg, Alemania) que toma mediciones con 50 frecuencias comprendidas en un rango de 5 a $1000 \mathrm{kHz}$. En 38 enfermos se repitió el análisis de bioimpedancia con el sistema BIVA al finalizar la hemodiálisis, utilizando los mismos pares de electrodos que se habían dejado puestos a lo largo de toda la sesión.

El monitor BIVA proporciona los datos de resistencia, reactancia y ángulo de fase a la frecuencia de $50 \mathrm{kHz}$. El monitor BIS proporciona la resistencia y el ángulo de fase para cada una de las frecuencias utilizadas. Para comparar los datos bioeléctricos de ambos monitores, utilizamos los resultados de resistencia y ángulo de fase del monitor BIS a la frecuencia de $50 \mathrm{kHz}$. Para analizar el estado de hidratación hemos asignado en el sistema BIVA una escala ordinal de siete puntos (de 0 a \pm 3 ) a lo largo del eje mayor de las tres elipses de tolerancia $(95 \%, 75 \%, 50 \%)$ desde el polo inferior de mayor hidratación al polo superior de menor hidratación, tal como propone Piccoli(1). Con el monitor BIS el estado de hidratación se determinó con el valor del exceso de hidratación prediálisis $(\mathrm{OH}$, "overhydratation") proporcionado por el monitor. EI $\mathrm{OH}$ postdiálisis se calculó restando al valor $\mathrm{OH}$ el volumen ultrafiltrado (OH PostHD).

Los estados de hidratación se definieron con los siguientes criterios. En el análisis con monitor BIS utilizamos dos criterios para definir el estado de hiperhidratación prediálisis: volumen $\mathrm{OH}$ superior al $15 \%$ del volumen de agua extracelular ${ }^{(13)}$ y volumen $\mathrm{OH}$ superior a 2.5 $\operatorname{litros}^{(14)}$. El estado de hidratación postdiálisis fue considerado de normohidratación cuando el $0 \mathrm{H}$ PostHD estaba en el rango de -1,1 a 1,1 litros; de hiperperhidratación si era superior a 1,1 litros, y de deshidratación cuando era inferior a -1.1 litros ${ }^{(15)}$. Con el monitor BIVA se consideró que el estado de hidratación era adecuado cuando el vector de impedancia se mantiene en el eje de hidratación dentro de la elipse de tolerancia del $75 \%$ tanto en los controles pre como postdiálisis ${ }^{(16)}$. Con arreglo a este criterio, se definió el estado de hiperhidratación prediálisis cuando el vector de impedancia en el estudio prediálisis estaba por debajo de la elipse de tolerancia del $75 \%$ (en la escala ordinal de hidratación corresponde a los valores $+3 y+2$ ). En el estudio postdiálisis se utilizó el mismo criterio para definir la hiperhidratación; el estado de normohidratación postdiálisis fue considerado cuando el vector de impedancia postdiálisis estaba dentro de la elipse de tolerancia del $75 \%$ (valores $+1,0$ y -1 de la escala ordinal) y se definió la deshidratación cuando el vector de impedancia postdiálisis estaba por encima de la elipse de tolerancia del 75 $\%$ (en la escala ordinal de hidratación corresponde a los valores -2 y -3$)^{(17)}$.

Análisis estadístico: Los resultados se expresan como media desviación estándar (DS). Los datos analizados tenían una distribución normal (test de KolmogorovSmirnov), por lo que se utilizaron tests paramétricos. La diferencia entre el valor de cada parámetro obtenido por los monitores BIVA y BIS en cada paciente, es el sesgo entre ambas medidas. Esta misma diferencia en valor absoluto expresada en porcentaje de la media aritmética de ambos valores (diferencia relativa), permite conocer la variabilidad entre las distintas mediciones. La correlación entre los diferentes métodos se hizo mediante el coeficiente de Pearson. Para categorías cuantitativas el análisis de concordancia se completó con el coeficiente de correlación intraclase (CCI) (18); el CCI varía entre 0 (ausencia de concordancia) y 1 (concordancia absoluta). Para categorías binarias y ordinales utilizamos respectivamente el índice kappa y el índice kappa ponderado(19); se considera que el nivel de concordancia es aceptable cuando el índice kappa es superior a 0.40 y excelente cuando es superior a 0.75. Para la comparación de medias se utilizó el test de Student y el test ANOVA según procediera. Los valores de $p<0,05$ se consideraron estadísticamente significativos.

\section{Resultados}

En la tabla 1 expresamos los datos de resistencia y ángulo de fase proporcionados por el monitor BIVA y por el monitor BIS a la frecuencia de $50 \mathrm{kHz}$, y los valores de los componentes corporales obtenidos con ambos monitores. Las cifras de la resistencia tienen una variabilidad mínima y el coeficiente de correlación intraclase indica que el nivel de concordancia intermétodo es casi absoluto. La medición del ángulo de fase con ambos monitores es diferente desde el punto de vista estadístico. Sin embargo su variabilidad es aceptable desde el punto de vista clínico $(11,5 \%)$ y el coeficiente de correlación intraclase de 0,92 indica una excelente concordancia intermétodo. 
Tabla 1. Cifras de Resistencia y Angulo de Fase con el monitor vectorial de monofrecuencia (BIVA), y con el monitor de multifrecuencia espectroscópica (BIS) a la frecuencia de corriente de $50 \mathrm{kHz}$, y valores de los volúmenes de agua corporal total (TBW), agua extracelular (ECW) y agua intracelular (ICW), de la masa grasa (FM) y de la masa celular (BCM) proporcionados por ambos monitores

\begin{tabular}{|c|c|c|c|c|c|c|c|}
\hline & BIVA & BIS & & $\begin{array}{l}\text { Diferencia } \\
\text { media (IC) }\end{array}$ & $\begin{array}{l}\text { Diferencia } \\
\text { relativa \% }\end{array}$ & $\begin{array}{c}\text { Coef } \\
\text { Pearson }\end{array}$ & CCI \\
\hline Resistencia (ohm) & $510,1 \pm 75.6$ & $515,4 \pm 78$ & $p=n . s$ & $\begin{array}{c}-5,3 \\
(-55,3,25,2)\end{array}$ & $1,3 \pm 1,7$ & $r=0,99$ & 0,99 \\
\hline Angulo fase $\left({ }^{\circ}\right)$ & $4,7 \pm 0,9$ & $4,2 \pm 1$ & $p<0,05$ & $\begin{array}{c}0,5 \\
(0,1,3)\end{array}$ & $11,5 \pm 6,5$ & $r=0,97$ & 0,92 \\
\hline TBW (I) & $38,7 \pm 7,8$ & $32,1 \pm 6,3$ & $p<0,001$ & $\begin{array}{c}6,6 \\
(2,6,17,8)\end{array}$ & $18,4 \pm 6,1$ & $r=0,95$ & 0,65 \\
\hline ECW (I) & $20,3 \pm 4,5$ & $16,2 \pm 3,3$ & $p<0,001$ & $\begin{array}{c}4,2 \\
(0,3,10,1)\end{array}$ & $22,5 \pm 9,1$ & $r=0,89$ & 0,55 \\
\hline ICW (I) & $18,1 \pm 4,6$ & $15,9 \pm 3,4$ & $p<0,01$ & $\begin{array}{c}2,2 \\
(-3,5,6,2)\end{array}$ & $13 \pm 6,3$ & $r=0,96$ & 0,80 \\
\hline FM (kg) & $22,1 \pm 6,2$ & $28,1 \pm 8,4$ & $p<0,001$ & $\begin{array}{c}-6 \\
(-19,2, \quad 0,8)\end{array}$ & $23,6 \pm 13$ & $r=0,91$ & 0,65 \\
\hline BCM (kg) & $22,8 \pm 6,5$ & $16,1 \pm 5,1$ & $p<0,001$ & $\begin{array}{c}6,7 \\
(-2,-18,2)\end{array}$ & $35,8 \pm 13,5$ & $r=0,86$ & 0,51 \\
\hline
\end{tabular}

IC: Intervalo de confianza (media $\pm 1,96 D S$ ). CCI: Coeficiente de correlación intraclase. El Coeficiente de Pearson mostró una correlación estadísticamente significativa en todos los parámetros analizados con una $p<0,001$

De los diferentes componentes corporales proporcionados por los dos monitores, solo el volumen de agua intracelular (ICW) muestra una variabilidad entre ambos monitores que puede ser asumida (13\%); en los restantes componentes el sesgo y la variabilidad son muy altos. Aunque el coeficiente de Pearson expresa una buena correlación entre las mediciones de ambos monitores, el coeficiente de correlación intraclase indica que el grado de concordancia es mediocre.

La tabla 2 muestra los parámetros del monitor BIS relacionados con el estado de hidratación $(\mathrm{OH}$ y el cociente $\mathrm{OH} / \mathrm{ECW}$ ) para los 7 valores de la escala ordinal que mide el nivel de hidratación en el monitor BIVA, y permite comprobar una buena correlación entre ambos procedimientos.

La clasificación de los enfermos según el estado de hidratación pre y postdiálisis está expresada en las tablas 3 y 4 . En la situación prediálisis el índice kappa para el diagnóstico de hiperhidratación es de 0,81 si el criterio diagnóstico de hiperhidratación con el sistema BIS fue $\mathrm{OH} / \mathrm{ECW}>0.15$ (concordancia excelente) y de 0,71 si el criterio diagnóstico fue $\mathrm{OH}>2,5$ I (concordancia aceptable). En la situación postdiálisis el índice kappa ponderado fue 0,64 (concordancia aceptable).
Tabla 2. Relación entre el grado de hidratación determinado por el el monitor vectorial de monofrecuencia (BIVA), y el exceso de hidratación $(\mathrm{OH})$ según el monitor de multifrecuencia espectroscópica (BIS)

\begin{tabular}{|c|c|c|}
\hline BIVA & \multicolumn{2}{|c|}{ BIS } \\
\hline & $0 H(1)$ & OH/ECW (\%) \\
\hline$+3(n=2)$ & $6,5 \pm 0,3$ & $31,8 \pm 5,1$ \\
\hline$+2(n=13)$ & $2,9 \pm 1,2$ & $16,7 \pm 5,9$ \\
\hline$+1(n=17)$ & $1,7 \pm 0,7$ & $9,9 \pm 3,8$ \\
\hline $0(n=15)$ & $1,1 \pm 0,9$ & $6,2 \pm 6$ \\
\hline$-1(n=4)$ & $0,3 \pm 0,3$ & $2,1 \pm 2,6$ \\
\hline$-2(n=2)$ & $-0,7 \pm 0,3$ & $-6,1 \pm 3,3$ \\
\hline$-3(n=1)$ & $-0,9$ & $-10,2$ \\
\hline
\end{tabular}

\section{Discusión}

Los diferentes fabricantes de monitores de bioimpedancia aseguran que sus procedimientos para calcular volúmenes y masas corporales están contrastados con los métodos de referencia, tanto en sujetos sanos como en pacientes afectos de diversas patologías, pero los re- 
[ Ruth Ros Nogales, et al ]

Bioimpedancia vectorial y espectroscópica: valoración del estado de hidratación con ambos métodos en hemodiálisis

Tabla 3. Estado de hidratación prediálisis. Concordancia entre los criterios de definición de los sistemas de bioimpedancia BIVA y BIS

\begin{tabular}{|c|c|c|c|}
\hline & $\begin{array}{c}\text { Hiperhidratado BIVA }(+3 \text { y }+2 \\
\text { de la escala ordinal) }\end{array}$ & $\begin{array}{c}\text { No hiperhidratado } \\
\text { BIVA (resto escala ordinal) }\end{array}$ & Total \\
\hline $\begin{array}{l}\text { Hiperhidratado BIS (criterio } \\
\text { OH/ECW>15\%) }\end{array}$ & 12 & 1 & 13 \\
\hline $\begin{array}{l}\text { No hiperhidratado BIS (crite- } \\
\text { rio } 0 \mathrm{H} / \mathrm{ECW} \leq 15 \% \text { ) }\end{array}$ & 3 & 38 & 41 \\
\hline Total & 15 & 39 & 54 \\
\hline Índice kappa & & & 0,81 \\
\hline $\begin{array}{l}\text { Hiperhidratado BIS (criterio } \\
\qquad \mathrm{OH}>2.5 \mathrm{I} \text { ) }\end{array}$ & 11 & 2 & 13 \\
\hline $\begin{array}{l}\text { No hiperhidratado BIS (crite- } \\
\text { rio } \mathrm{OH} \leq 2.5 \mathrm{I} \text { ) }\end{array}$ & 4 & 37 & 41 \\
\hline Total & 15 & 39 & 54 \\
\hline Índice kappa & & & 0,71 \\
\hline
\end{tabular}

Tabla 4. Estado de hidratación postdiálisis. Concordancia entre los criterios de definición de los sistemas de bioimpedancia BIVA y BIS

\begin{tabular}{|c|c|c|c|c|}
\hline & $\begin{array}{l}\text { Hiperhidratado } \\
\text { BIVA ( }+3 \text { y }+2 \text { de la } \\
\text { escala ordinal) }\end{array}$ & $\begin{array}{l}\text { Normohidratado } \\
\text { BIVA (+1, } 0 \text { y }-1 \text { de la } \\
\text { escala ordinal) }\end{array}$ & $\begin{array}{c}\text { Deshidratado BIVA } \\
\text { (-2 y }-3 \text { de la escala } \\
\text { ordinal) }\end{array}$ & Total \\
\hline $\begin{array}{l}\text { Hiperhidratado BIS } \\
(0 \mathrm{H} \text { postHD > } 1.1 \mathrm{I})\end{array}$ & 2 & 2 & 0 & 4 \\
\hline $\begin{array}{c}\text { Normohidratado BIS } \\
\text { (OH PostHD de }-1.1 \mathrm{a} \\
1.1 \mathrm{I})\end{array}$ & 2 & 16 & 0 & 18 \\
\hline $\begin{array}{l}\text { Deshidratado BIS (OH } \\
\text { PostHD <-1.1 I) }\end{array}$ & 0 & 5 & 11 & 16 \\
\hline Total & 4 & 23 & 11 & 38 \\
\hline Índice kappa & & & & 0,64 \\
\hline
\end{tabular}

sultados obtenidos con los diversos sistemas de bioimpedancia muestran una gran variablidad interméto-

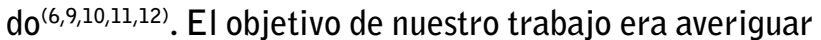
si la variabilidad intermétodo es debida a la diferente lectura de los parámetros bioeléctricos 0 a las ecuaciones utilizadas por cada monitor para la cuantificación de los compartimentos corporales.

Las mediciones de resistencia y ángulo de fase, realizadas por los monitores BIVA y BIS a la frecuencia de $50 \mathrm{kHz}$, tienen un alto grado de concordancia. La variabilidad media para la resistencia es sólo del 1,3\%, similar a la variabilidad intraindividuo ${ }^{(3,16)}$. El coeficiente de correlación intraclase $(0,99)$ indica que la concordancia de ambos monitores es prácticamente absoluta. Las mediciones del ángulo de fase son diferentes desde el punto de vista estadístico, pero la variabilidad me- dia $(11,5 \%)$ puede ser asumible desde el punto de vista clínico y el coeficiente de correlación intraclase $(0,92)$ corresponde a un alto grado de concordancia. Podemos concluir que ambos monitores realizan unas mediciones muy parecidas de los parámetros bioeléctricos a la frecuencia de $50 \mathrm{kHz}$.

Las mediciones de los volúmenes de agua corporal total, agua extracelular, agua intracelular, masa grasa y masa celular tienen un sesgo y una variabilidad alta. Igual que otros estudios previos realizados con los mismos monitores ${ }^{(10,11)}$, hemos comprobado que el monitor BIVA proporciona valores más altos que el monitor BIS para todos los compartimentos analizados menos para la masa grasa. La mejor concordancia corresponde al volumen de agua intracelular (sesgo medio 2,2 litros, variabilidad media $13 \%$, coeficiente de correlación in- 
traclase de 0,80$)$ y puede ser aceptable. Para el resto de los compartimentos el sesgo y la variabilidad no son asumibles, y el coeficiente de correlación intraclase indica que la equivalencia es mediocre. El coeficiente de Pearson demuestra que hay una correlación estrecha en los resultados de ambos modelos, pero no es un test válido para un estudio de concordancia $(18,19,20)$. La mayoría de las ecuaciones que cuantifican las masas y volúmenes corporales solo utilizan la resistencia como parámetro bioeléctrico ${ }^{(1,3,5)}$. Como el nivel de concordancia en la lectura de la resistencia a la frecuencia de $50 \mathrm{kHz}$ fue prácticamente absoluto, tenemos que asumir que la variabilidad intermétodo debe ser atribuida a los diferentes modelos bioeléctricos y ecuaciones utilizadas en cada sistema de bioimpedancia.

Además de cuantificar los volúmenes y masas corporales, los distintos sistemas de bioimpedancia tienen criterios para clasificar a los enfermos según el estado de hidratación. El sistema BIS utiliza en el momento prediálisis el parámetro $\mathrm{OH}$ expresado en litros ${ }^{(14)} \mathrm{O}$ en porcentaje del volumen de agua extracelular(13) y para el momento postdiálisis el valor en litros del parámetro $\mathrm{OH}$ postdiálisis estimado ${ }^{(15)}$. El sistema BIVA define los estados de hidratación pre y postdiálisis aplicando una escala ordinal a las elipses de tolerancia ${ }^{(1,17)}$. Cuando estudiamos la equivalencia de ambos sistemas para clasificar a los enfermos según su estado de hidratación, hemos comprobado que el grado de concordancia era bueno tanto para definir el estado de hiperhidratación prediálisis como los estados de hiper, normo o deshidratación postdiálisis. Aunque los resultados de los diferentes compartimentos del agua corporal obtenidos con ambos monitores no son intercambiables, los criterios usados para definir el estado de hidratación muestran un alto grado de coincidencia en la clasificación de los enfermos.

El ángulo de fase es un parámetro bioeléctrico relacionado con la nutrición, y tiene valor pronóstico en los enfermos con insuficiencia renal ${ }^{(21,22,23,24)}$. En su valoración hay que tener en cuenta que el ángulo de fase varía con el estado de hidratación ${ }^{(25,26)}$ y aumenta tras la sesión de hemodiálisis ${ }^{(16,27)}$. Nuestro estudio indica que en el periodo prediálisis, los dos monitores tienen un grado aceptable de concordancia y que el valor del ángulo de fase obtenido con cualquiera de ellos puede tener la misma significación para su utilización en un análisis de pronóstico o en un estudio de nutrición.

Concluimos que los monitores de los sistemas BIVA y BIS que hemos utilizado proporcionan datos equiparables de la resistencia y del ángulo de fase a la frecuen- cia de 50 kHz. La cuantificación de los compartimentos corporales tiene una alta variabilidad intermétodo que debe ser atribuida a las ecuaciones utilizadas. Sin embargo los diferentes criterios para definir los estados de hidratación con ambos sistemas, son concordantes y clasifican a los enfermos de forma bastante homogénea.

La elección del sistema de bioimpedancia a utilizar en los enfermos dializados, es motivo de gran controversia. Con los datos del presente trabajo no se puede deducir cuál de los dos sistemas es más aconsejable. No obstante, si utilizamos el ángulo de fase y el volumen de agua intracelular como parámetros nutricionales y analizamos el grado de hidratación tal como lo hemos hecho nosotros, los resultados de ambos sistemas son intercambiables, y en nuestra opinión los dos procedimientos tienen la misma utilidad clínica.

\section{AGRADECIMIENTOS}

Al Dr. Teruel Briones (Jefe de Seccion de la Unidad de Hemodiálisis) por su ayuda, y colaboración sin ella no habria sido posible su realización, asi como a la Dra. Fernandez Lucas (Medico Adjunto de la Unidad de Hemodiálisis), y a nuestros compañeros.

Recibido: 14 Enero 2013

Revisado: 20 Enero 2013

Modificado: 11 Febrero 2013

Aceptado: 14 Febrero 2013

\section{Bibliografía}

1. Piccoli A, Nescolarde LD, Rosell J: Análisis convencional y vectorial de bioimpedancia en la práctica clínica. Nefrología 2002; XXII: 228-238.

2. Cooper BA, Aslani A, Ryan M, Zhu FYP, Ibels LIS, Allen BJ, Pollock CA: Comparing different methods of assessing body omposition in end-stage renal failure. Kidney Int 2000; 58: 408-416.

3. Kyle UG, Bosaeus I, De Lorenzo AD, Deurenberg P, Elia M, Gómez JM, Heitmann LB, Kent-Smith L, Melchior JC, Pirlich M, Scharfetter H, Schols AMWJ, Pichard C, Composition of the ESPEN Working Group: Bioelectrical impedance analysispart I: review of principles and methods. Clin Nutr 2004; 23: 1226-1243. 
4. Kaysen GA, Zhu F, Sarkar S, Heymsfield SB, Wong $J$, Kaitwatcharachai Ch, Kuhlmann MK, Lvin NW: Estimation of total-body and limb muscle mass in hemodialysis patients by using multifrequency bioimpedance spectroscopy. Am J Clin Nutr 2005; 82: 988-995.

5. Moissl UM, Wabel P, Chamney PW, Bosaeus I, Levin NW, Bosy-Westphal A, Korth 0, Müller MJ, Ellegard L, Malmros V, Kaitwatcharachai C, KuhImann MK, Zhu F, Fuller NJ: Body fluid volume determination via body composition spectroscopy in health and disease. Physiol Meas 2006: 27: 921-933.

6. Jaffrin MY, Morel H: Body fluid volumes measurements by impedance: A review of bioimpedance spectroscopy (BIS) and bioimpedance analysis (BIA) methods. Med Eng Phys 2008; 30: 12571269.

7. López-Gómez JM: Evolución y aplicaciones de la bioimpedancia en el manejo de la enfermedad renal crónica. Nefrología 2011; 31: 630-634.

8. Cigarrán Guldris S: Aplicaciones futuras de la bioimpedancia vectorial (BIVA) en nefrología. 2011; 31: 635-643.

9. Donadio C, Consani C, Ardini M, Bernabini G, Caprio $F$, Grassi $G$, Luccchesi $A$, Nerucci $B$ : Estimate of body water compartments and of body composition in maintenance hemodialysis patients: Comparison of single and multifrequency bioimpedance analysis. J Ren Nutr 2005; 15: 332-344.

10. Arias $M$, Massó $E$, Fontseré $N$, Vera $M$, Durán $C$, Martina MN, Pérez NS, Maduell F: ¿Son comparables los métodos de bioimpedanciometría utilizados en hemodiálisis en la actualidad?. Nefrología 2010; 30 (Supl 1): 88 (abstract).

11. Dou Y, Liu L, Cheng X, Cao L, Zuo L: Comparison of bioimpedance methods for estimating total body water and intracellular water changes during hemodialysis. Nephrol Dial Transplant 2011; 26: 3319-3324.

12. Wabel $P$, Chamney $P$, Moiss $U$, Jirka T: Importance of whole-body bioimpedance spectroscopy for the management of fluid balance. Blood Purif 2009; 27: 75-80.
13. Passauer J, Petrov H, Schleser A, Leicht J, Pucalka K: Evaluation of clinical dry weight assessment in haemodialysis patients using bioimpedance spectroscopy: a cross-sectional study. Nephrol Dial Transplant 2010; 25: 545-551.

14. Piccoli A, for the Italian HD-BIA Study Group: Identification of operational clues to dry weight prescription in hemodialysis using bioimpedance vector analysis. Kidney Int 1998; 53: 1036-1043.

15. Guida B, De Nicola L, Trio R, Pecoraro P, Iodice C, Memoli B: Comparison of vector and conventional bioelectrical impedance analysis in the optimal dry weight prescription in hemodialysis. Am J Nephrol 2000; 20: 311-318.

16. Hernández Aguado I, Porta Serra $M$, Miralles $M$, García Benavides F, Bolumar F:La cuantificación de la variabilidad en las observaciones clínicas. Med Clin (Barc) 1990: 95: 424-429.

17. Bland JM, Altman DG: Statistical methods for assessing agreement between two methods of clinical measurement. Lancet i: 307-310, 1986.

18. Fiedler R, Jehle PM, Osten B, Dorligschaw 0, Girn$\mathrm{dt} M$ : Clinical nutrition scores are superior for the prognosis of haemodialysis patients compared to lab markers and bioelectrical impedance. Nephrol Dial Transplant 2009; 24: 3812-3817.

19. Segall $L$, Mardare NG, Ungureanu $S$, Busuioc $M$, Nistor I, Enache R, Marian S, Covic A: Nutritional status evaluation and survival in haemodialysis patients in one centre from Romania. Nephrol Dial Transplant 2009; 24: 2536-2540.

20. 24.- Abad $S$, Sotomayor $G$, Vega $A$, Pérez de José $A$, Verdalles $U$, Jofré $R$, López Gómez JM: El ángulo de fase de la impedancia elécrica es un predictor de supervivencia a largo plazo en pacientes en diálisis. Nefrología 2011; 31: 670-676.

21. Piccoli $A$, for the Italian CAPD-BIA Study Group: Bioelectric impedance vector distribution in peritoneal dialysis patients with different hydratation status. Kidney Int 2004; 65: 1050-1063.

22. Valdés Sotomayor J, Chust Alvarez $M$, Navarro Martínez V, Morate Rostra L, Escuin Fernández R, Castillo Garrote A, Giera Vives L, Onieva Martell 
C: El estado nutricional según bioimpedancia varía dependiendo del estado de hidratación. Nefrología 2010; 30 (Supl 1): 97 (abstract).

23. Giorgi $M$, González Lázaro $G$, Cigarrán $S$, Lorenzo A, Salanova L, Sánchez Tomero JA, Barril G: Dif- erencias en la composición corporal pre y post HD medidas por bioimpedancia vectorial y su correlación con parámetros bioquímicos y $\mathrm{K}$ corporal total. Nefrología 2009; 29 (Supl 2): 90 (abstract). 